\title{
Vector coding in slow goal-directed arm movements
}

\author{
J. B. DE GRAAF \\ Delft University of Technology, Delft, The Netherlands \\ J. J. DENIER VAN DER GON \\ University of Utrecht, Utrecht, The Netherlands \\ and \\ A. C. SITTIG \\ Delft University of Technology, Delft, The Netherlands
}

\begin{abstract}
It has been found that the estimate of relative target direction is consistently biased. Relative target direction refers to the direction in which a target is located relative to another location in space (e.g., a starting position in the case of goal-directed movements). In this study, we have tested two models that could underlie this biased estimate. The first proposed model is based on a distorted internal representation of locations (i.e., we perceive a target at the "wrong" location). We call this the distorted location model. The second model is based on the idea that the derivation of target direction from spatial information about starting and target position is biased. We call this the biased direction model. These two models lead to different predictions of the deviations that occur when the distance between the starting position and the target position is increased. Since we know from previous studies that the initial direction of slow arm movements reflects the target direction estimate, we tested the two models by analyzing the initial direction of slow arm movements. The results show that the biased direction model can account for the biases we find in the target direction estimate for various target distances, whereas the distorted location model cannot. In two additional experiments, we explored this model further. The results show that the biases depend only on the orientation of the line through starting position and target position relative to the plane through longitudinal head or body axis and starting position. We conclude that the initial part of (slow) goal-directed arm movements is planned on the basis of a (biased) target direction estimate and not on the basis of a wrong internal representation of target location. This supports the hypothesis that we code displacements of our limbs in space as a vector.
\end{abstract}

When subjects are instructed to move an arm slowly and accurately in the direction of a target, they start the arm movement in a direction that consistently deviates from target direction (de Graaf, Sittig, \& Denier van der Gon, 1991, 1994). Figure 1 shows a typical example of the curved movement trajectories of slow goal-directed arm movements that we found in those studies. One could think that the deviations in initial movement directions are caused by biomechanical constraints of the arm. However, this explanation is highly unlikely. First, when subjects perform the same task in a vertical plane, we see similar deviations in initial movement direction in spite of different forces acting on the arm (de Graaf, 1994). Second, if subjects are not to move the hand toward a target but are explicitly instructed to move the hand in a straight

The authors wish to thank J. B. J. Smeets and E. Brenner for the interesting discussions about the models. The authors are also grateful to anonymous referees for useful comments and to Sheila McNab for improving the language and clarity of the paper. Correspondence should be addressed to J. B. de Graaf, C.H.R.U. Pontchaillou, Clinique Neurologique, Rue Henri Guilloux, 35033 Rennes, France (e-mail: degraaf@ arles.univ-rennes 1.fr). line, they are very well able to do so (de Graaf et al., 1994). Third, it is not only in motor tasks that such deviations occur. If subjects are instructed to indicate the perceived direction of targets by using a pointer (de Graaf et al., 1991) or in a three-dot alignment task (Sittig \& de Graaf, 1994), similar direction-dependent deviations are found, although now the motor component of the task is negligible.

Apparently, we can rule out the output modality of the task (i.e., biomechanical factors) as a possible cause of the deviations. Are the deviations then related to the input modality? In most experiments, the targets were presented visually. Could the deviations in initial movement direction originate from imperfections or peculiarities in the processing of the visual information of the target? This is also highly unlikely, since congenitally blind and blindfolded sighted subjects show similar biases when moving the hand toward targets located by touch (de Graaf et al., 1994) or directing a pointer (Hollins \& Kelley, 1988).

These results indicate that the occurrence of these small but very consistent biases in directing tasks is not restricted to spatial information processing of one specific sensor modality: the deviations occur not only in a purely visual tasks (such as the pointer-setting tasks) but also in a 


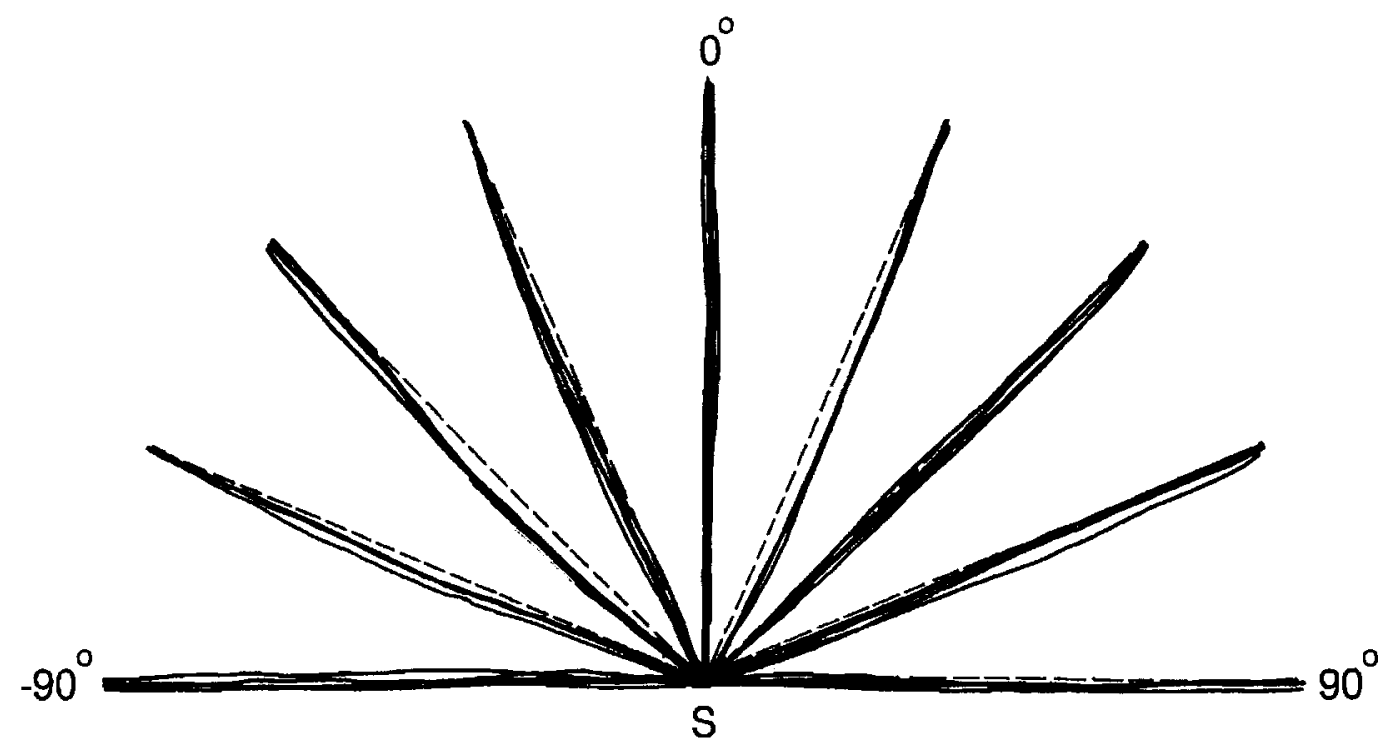

\begin{abstract}
Figure 1. Superimposed recordings of the movement trajectories of 1 subject. The subject was seated behind a horizontal table with the starting position (S) in the medial plane at a distance of $26 \mathrm{~cm}$ from the body. His eyes were $45 \mathrm{~cm}$ above the stimulus surface. The subject was instructed to move his right arm slowly and accurately from $S$ in the direction of the visual targets ( $31 \mathrm{~cm}$ from S). Note the systematic deviations from the straight line (indicated by dashed lines) between $S$ and target position for most target positions.
\end{abstract}

purely kinesthetic task (such as with the blind and blindfolded subjects). Also, the deviations are not restricted to one specific output modality: they occur in a motor task as well as in a visual adjustment task. The deviations seem to be an expression of a more general phenomenon of spatial perception.

In this paper, we investigate two distinct models that could underlie the biases in directional tasks. Since, as argued above, the deviations in initial movement direction seem to originate from the processing of spatial information before the actual start of the movement, we pay special attention to the very first part of the movement trajectories. This stage of a movement best reflects the information available before the internal representation is updated during the movement.

\section{MODELS}

\section{Distorted Location Model}

One possible explanation for the biases in directional tasks is that we perceive starting and target positions at a "wrong" location. If this is the case, then indicating the perceived direction by pointing or by moving toward the target could result in consistent deviations. This model is based on the notion that the trajectory of a goal-directed movement is planned as a straight line through starting and target position in the internal representation. The actual movement trajectory (i.e., the trajectory along which the subject moves the hand when performing the task) is then the straight internal path that is transformed back into the physical world. The notion of an internal path of the movement that is generated to control the movement has been proposed before (Van Sonderen \& Denier van der Gon, 1990; Vincken \& Denier van der Gon, 1985).

The transformation of locations between the real world and the internal representation must be nonlinear in order to explain the demonstrated biases. If the transformation were linear, straight internal paths would be transformed into actual straight movement paths, which would result in correct initial movement directions for all target positions. To illustrate that a nonlinear transformation between locations in the real world and locations in the internal representation can indeed result in the deviations in initial movement direction such as we found before, we will give an example: One kind of transformation that results in the curved trajectories such as we found (see Figure 1) is a nonlinear contraction of locations toward the frontal plane and parallel to the medial plane of the subject. A contraction of space has been proposed by Hollins and Kelley (1988). In Figure 2A, such a transformation between the real world and the internal representation is drawn schematically. Note that if an object is farther from the frontal plane of the subject, it is disproportionally more displaced toward the frontal plane. To examine the effects of such a contraction, we calculated the predicted deviation in initial movement direction for a representative contraction. Figure $2 \mathrm{~B}$ shows these predicted deviations as well as a typical example of the deviations in initial movement direction. It is clear that the patterns are fairly similar. This illustrates that a nonlinear transformation between locations in the real world and locations in the internal representation can indeed result in deviations in initial movement direction such as those we found in previous research. 

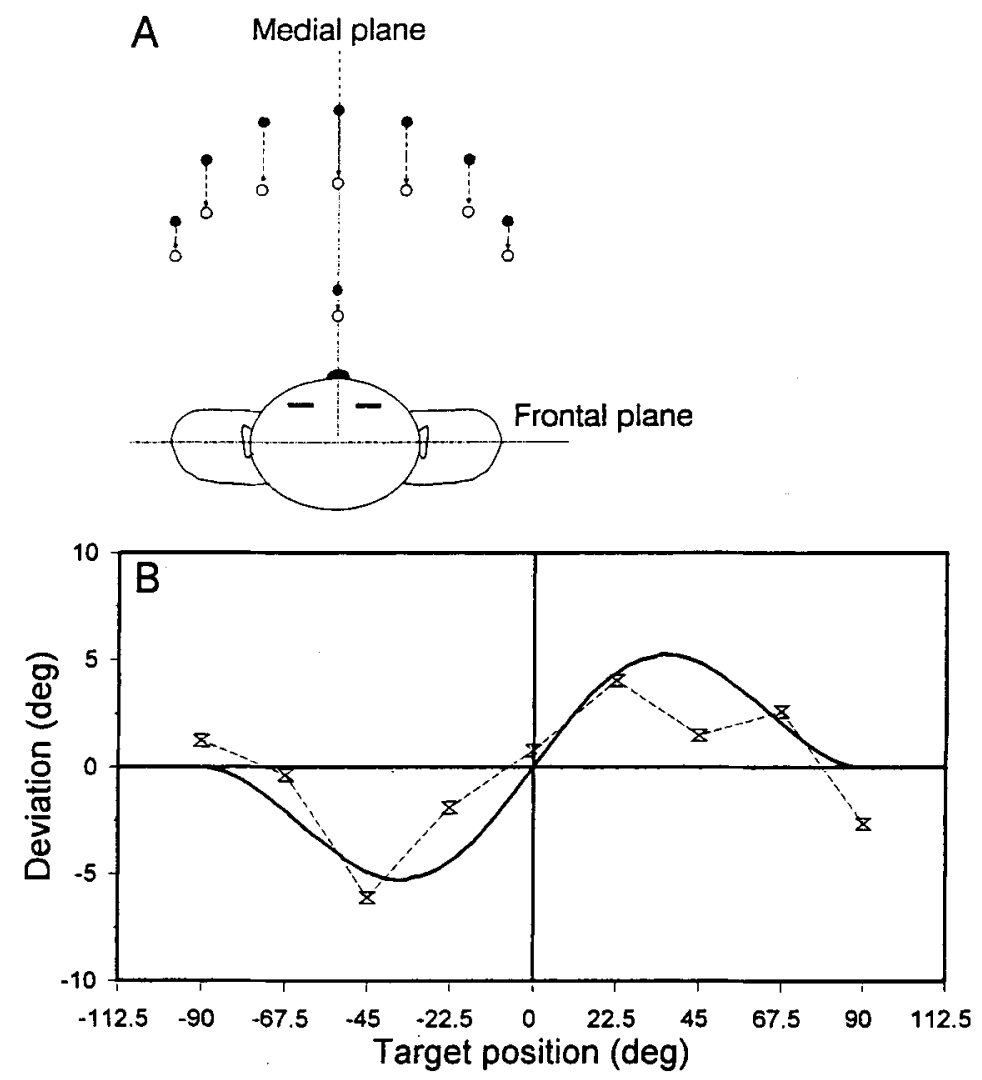

Figure 2. A specific example of a distorted location model: a nonlinear contraction. (A) Effect on locations of a nonlinear contraction parallel to the medial plane of the subject, drawn schematically. Black dots represent location in the real worid; white dots represent "location" in the internal representation. Note that the contraction is disproportionally stronger the farther the locations are from the body. (B) A rather arbitrarily chosen example of a nonlinear contraction. Predicted deviations in initial movement direction as a function of target direction (solid curve), and the results for the subjects whose trajectories are depicted in Figure 1 (dashed curve). The transformation of locations from the real world to locations in the internal representation is chosen here to be $\emptyset(x, y)=(x, a \sqrt{ } y)$, with $a=2.5$. The inverse transformation then is $\emptyset^{-1}(u, v)=\left(u, v^{a}\right)$. In the model, as well as in the experiment, the starting position was located $26 \mathrm{~cm}$ in front of the subject and the targets were $31 \mathrm{~cm}$ from the starting position.

What deviations does a distorted location model in general predict for other starting and target positions? Although one obviously cannot give a quantitative prediction for every type of distortion, one can give a qualitative prediction: Any distorted location model will predict an increase in the deviations with increasing distance between starting and target position. In the Appendix, we prove this for an arbitrary transformation that fits in with the results we have previously found (see also the Discussion section). In Figure 3, we illustrate the qualitative prediction schematically. Figure $3 \mathrm{~A}$ shows locations in the real world, and Figure 3B shows locations in the internal representation. If, in the real world, two targets are located at different distances but are on the same straight line from the starting position (dashed line in Figure 3A), then because of the nonlinear transformation, in the internal representation, these targets are located on some curve through the starting position (dashed curve in Fig- ure 3B). In the internal representation, the two target positions are not on the same straight line through the starting position (solid lines in Figure 3B); therefore, in the real world, the two curved movement trajectories are not identical (solid curves in Figure 3A). The movement trajectory for the target that is located farther from the starting position is more curved and thus will show larger deviations in initial movement direction. For the specific example given in Figure 2, which is a transformation that predicts the deviations we have previously found, it can be calculated that bringing the target distance from 31 to $62 \mathrm{~cm}$ will approximately double the magnitude of the deviations in initial movement direction.

\section{Biased Direction Model}

Another possible explanation for the deviations in initial movement direction is based on the idea that we do not code the displacements of our limbs in space in terms 

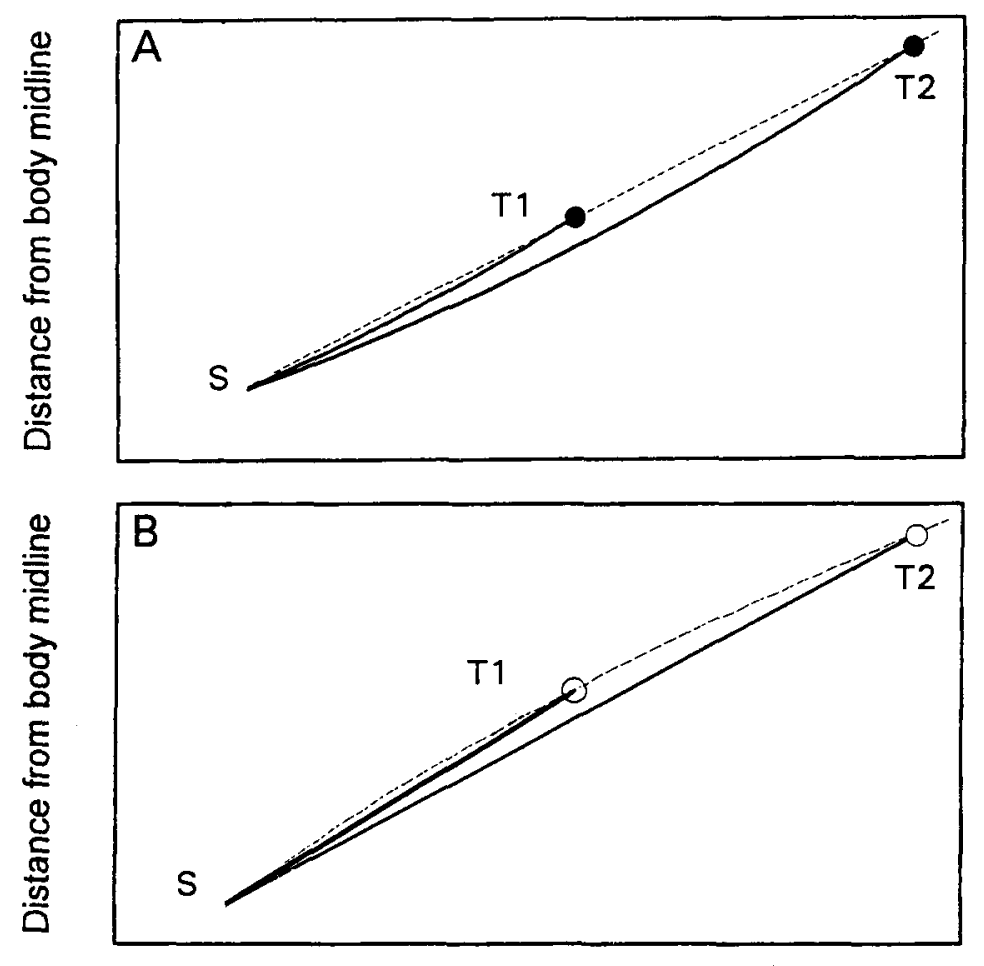

Distance from medial plane

Figure 3. Prediction of a general distorted location hypothesis about the magnitude of deviations in initial movement direction for increasing distance between starting position and target position. Dashed lines indicate the straight line through starting position and target position in the real world (In Panel $A$ ) and its image in the internal representation (in Panel B). Solid lines indicate straight lines through the internally represented target positions (in Panel B) and the actual movement trajectories in the real world (in Panel $A$ ).

of successive locations, as was the case in the distorted location model, but rather we encode them in terms of a vector (e.g., Paillard, 1991a). If the process of deducing information about target direction relative to the starting position leads to a biased estimate, then the initial direction of limb movements can indeed deviate from target direction. This explanation is essentially different from the distorted location model, since even if the internal representation of locations is correct, deviations can occur. Because, in this biased direction model, information about the direction of the target is uncoupled from information about the distance of the target, it follows rather naturally that in this model the biases in the direction estimate depend solely on the direction of the target relative to the starting position (i.e., on the orientation of the line through starting position and target position relative to a certain reference). Therefore, this model predicts that the deviations are independent of the distance between starting position and target position. Note that this prediction is different from that of the distorted location model.

The idea that, in this model, the directional biases are independent of the distance of the target relative to the starting position is in fact the same as stating that the process underlying the directional biases is a linear (i.e., scale-independent) process. Note that, in the distorted location model, the transformation needed to be nonlinear, which, as we saw, implies that the process is not scaleindependent. This shows the essential difference between the two models: In the biased direction model, we have no (back) transformation from locations in the internal representation into locations in the real world, since the locations in the internal representation are not used to plan successive locations of the movement trajectory in the real world but are used to deduce information about the direction (and the distance) of the target relative to the starting position. The resulting movement in the real world starts exactly in this direction.

In this study, ${ }^{1}$ we have tested the distorted location model against the biased direction model by varying the distance between starting and target position (Experiment 1 ) and by analyzing the first part of the movement trajectories. The results show that the deviations in initial movement direction are not influenced by the distance between starting and target position, which supports the biased direction model. To further explore this model, we performed two experiments in which we varied the location of the starting position. In Experiment 2, 
we varied the distance between the whole stimulus configuration (i.e., starting and target positions) and the body of the subject. In Experiment 3, we translated the starting position to the right of the medial plane of the subject. The results show that the estimate of target direction depends on the line through starting and target position relative to the plane through the longitudinal head or body axis and starting position.

\section{METHOD}

\section{Subjects}

Eleven subjects participated in this study ( 4 male, 7 female, age range $=22-65$ years), with a subset of them participating in each experiment. All subjects were right-handed and had normal or corrected-to-normal vision. Two subjects were informed about the purpose of the study. The results of these 2 subjects did not differ from the results of the other subjects. Also, the results of the oldest subject did not differ from that of the others.

\section{Apparatus}

In all three experiments, the subjects were seated in front of a horizontal table (stimulus surface). The eyes of the subjects were approximately $45 \mathrm{~cm}$ above the surface (except in Condition 2 of Experiment 2). Light-emitting diodes (LEDs) were positioned under the stimulus surface to indicate the starting $(\mathrm{S})$ and target $(\mathrm{T})$ positions. The stimulus surface was made of a thin frosted screen so that the LEDs could not be located by touch and were visible only when they were lit. In several experimental conditions, we wanted the subjects to have no visual feedback of the moving arm. Therefore, in these conditions, a shield was placed between the head of the subject and the stimulus surface, such that the subject could see the target LEDs but not the arm. The shield was such that it excluded vision of the starting position and of the whole arm, including the shoulder. The presentation of starting and target positions was computer controlled. Their locations differed in the three experiments and will be given later.

In all experimental conditions, the movements were recorded with an Optotrak 2010 system. This system measures the threedimensional coordinates of an infrared LED with an accuracy of $0.5 \mathrm{~mm}$ in all directions. This infrared LED was attached to the nail of the index finger on the hand of the moving arm or to the tip of the stick (in Condition 2 of Experiments 2 and 3 ) and its position was sampled at $100 \mathrm{~Hz}$.

\section{Procedure}

The subjects were instructed to move the index finger or the tip of a stick slowly and accurately from the starting position in the direction of the target position. They were asked not to lift the finger or the tip of the stick from the table and to stop the movement at a radial distance of approximately $15 \mathrm{~cm}$ from the starting position. This was far enough since we were particularly interested in the first part of the movement trajectories. Moreover, in certain conditions, it prevented the subject from seeing the arm near the edge of the shield, and, in Experiment 2, the starting position was sometimes so far from the subject's body that he/she could move the arm over only a short distance toward the target position. The results of earlier experiments have shown that the instruction to move only a short distance toward the target has no influence on the initial movement direction, compared with the instruction to end a movement on the target. We will come back to this point in the Results section of Experiment 1.

In all three experiments, the subject was allowed to move his/her eyes and orient his/her head toward starting and target positions. The subject was asked not to move his/her body. The experimenter watched the subject to make sure that he/she did not do this. Before starting the experiments, 5-10 practice trials were given to all subjects in order to make them familiar with all of the experimental requirements.

A trial took the following form. The subject placed the index finger or the tip of the stick on the starting LED. Then, in the conditions where the subject had no visual feedback of the moving arm, the shield was placed between the eyes of the subject and the stimulus surface. A target position was chosen quasi-randomly from one of the corresponding target positions. The target LED remained visible for $10 \mathrm{sec}$, in which time the subject moved the hand or the tip of the stick from the starting position roughly $15 \mathrm{~cm}$ in what he/she thought was the direction of the target position. When the target LED went out, the shield was removed and the subject placed the index finger back on the starting. LED. In the conditions in which no shield was placed, the starting LED went out as soon as a target LED appeared, and the starting LED appeared again when the target LED went out.

All target positions were presented five times ( 10 times for 3 subjects in Condition 2 of Experiment 2). In some of the trials, the subjects reported that the movement had started in the wrong direction (which was mainly caused by unexpected jerky movement due to friction between the table and the finger). These trials were not included in the analysis. It turned out that in each condition for a given target position no more than one trial had to be excluded from the analysis.

\section{Analysis}

A detailed description and the rationale of the analysis can be found in de Graaf et al. (1994). In short, we determined the deviation in initial movement direction as follows. We used the raw position data for the index finger or the tip of the stick being between 2 and $4 \mathrm{~cm}$ radial distance from the starting position, and we determined the straight line through these data points by a least squares fit. Since the subjects moved with a velocity of $3-6 \mathrm{~cm} / \mathrm{sec}$ and the sampling rate was $100 \mathrm{~Hz}, 30-70$ data points were used to determine the straight line. The orientation of the resulting line is what we call the initial movement direction. (Because in many trials the onset of the movements showed some irregularities, possibly due to friction, we did not use the position data for the first $2 \mathrm{~cm}$ of the movements to determine the initial movement direction.) The deviation in initial movement direction is the angle between this line and the line between the point on this line at a radial distance of $3 \mathrm{~cm}$ from the starting position and the target position. A clockwise deviation is a positive deviation in initial movement direction. A deviation in initial movement direction could be determined within an accuracy of $2^{\circ}$.

\section{EXPERIMENTS AND RESULTS}

\section{Experiment 1: Increasing the Distance Between Starting Position and Target Position}

Experimental set-up. In Experiment 1, the relation between the distance between the starting position and the target position and the magnitude of the deviations was investigated. Seven subjects participated. Figure 4 shows the experimental set-up. The starting position was $26 \mathrm{~cm}$ in front of the subject. Seven targets were presented at a distance of $31 \mathrm{~cm}$, and seven targets at a distance of $62 \mathrm{~cm}$. Five subjects could see the moving arm. One of these subjects, as well as 2 other ones, participated in an additional experiment in which vision of the moving arm was prevented by a shield.

Results. Before showing the complete results of this experiment, we will first show that the instruction to move only $15 \mathrm{~cm}$ in the direction of the target did not change the initial direction of the movements relative to when sub- 


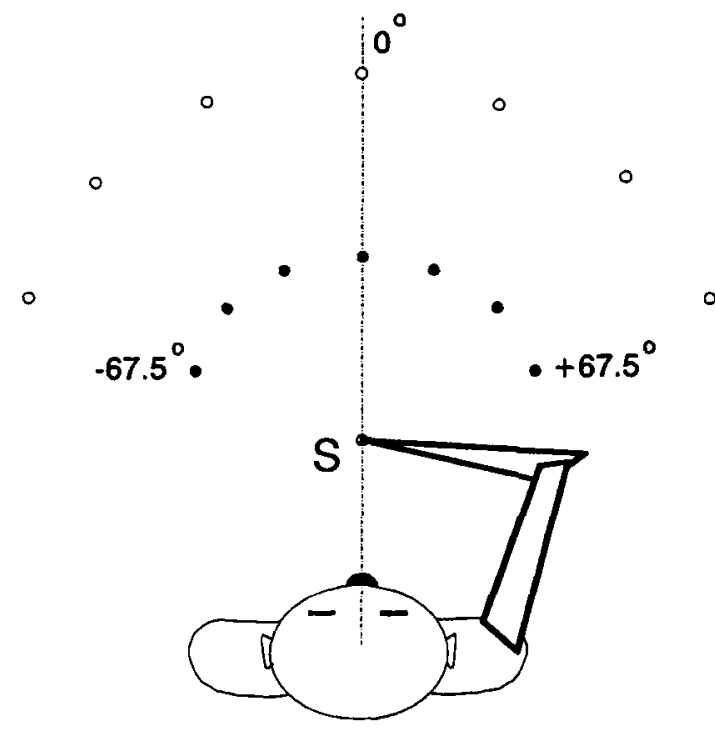

Figure 4. Top view of the experimental set-up for Experiment 1. In this figure, as well as in the following figures, target positions are represented by the angle between the saggital plane of the subject in which the starting position (S) was located and the line from $S$ to the target positions. Target positions have clockwise orientation. $S$ is presented at a distance of $26 \mathrm{~cm}$ straight ahead of the subject. Targets were presented at distances of $31 \mathrm{~cm}$ (black dots) and $62 \mathrm{~cm}$ (white dots) from $S$. The target positions ranged from $-67.5^{\circ}$ to $+67.5^{\circ}$, with intervals of $22.5^{\circ}$. The subject's eyes were at $45 \mathrm{~cm}$ above the stimulus surface.

jects completed the movement toward the target. In Figure 5, we plotted the deviations in initial movement direction we found in the present experiment and the deviations we found in a previous study (de Graaf et al., 1994), where subjects moved over the complete distance toward the target. In the two experiments, we used the same targets at $31 \mathrm{~cm}$ from the starting position. It can be seen that the deviations in initial movement direction (which were calculated in the same way) are similar for both movement distances. Statistical testing confirmed this observation $[F(1,8)=0.094, p>.75]$. This finding need not surprise us, since we already stated in the introduction that the deviations in initial movement direction reflect a process before the actual start of the movement, and, therefore, the actual movement distance is not likely to influence the magnitude of the deviations.

Figure 6 shows mean deviations in initial movement direction as a function of target position for 7 subjects. Figure 6A shows the results for a distance of $31 \mathrm{~cm}$ between the starting position and the target position, and Figure 6B shows the results for a distance of $62 \mathrm{~cm}$. Solid lines indicate trials where the subjects could see the moving arm; dashed lines indicate where they could not. (Note that 1 subject participated in both conditions.) First, it can be seen that the subjects showed clear deviations from target direction, away from the medial plane. These deviations in initial movement direction closely resemble those we have found in our previous studies (de Graaf et al., 1991, 1994). Second, it can be seen that the distance between the starting position and the target position had no clear influence on the initial direction of the movements. This holds both for trials where the subjects could see the moving arm and for trials where they could not. Figure $6 \mathrm{C}$ shows the difference between the deviations in initial movement direction for movements directed at the $31-\mathrm{cm}$ targets and the deviations for movements directed at the $62-\mathrm{cm}$ targets. According to the distorted location model, this difference should be positive for targets on the right side of the stimulus sur-

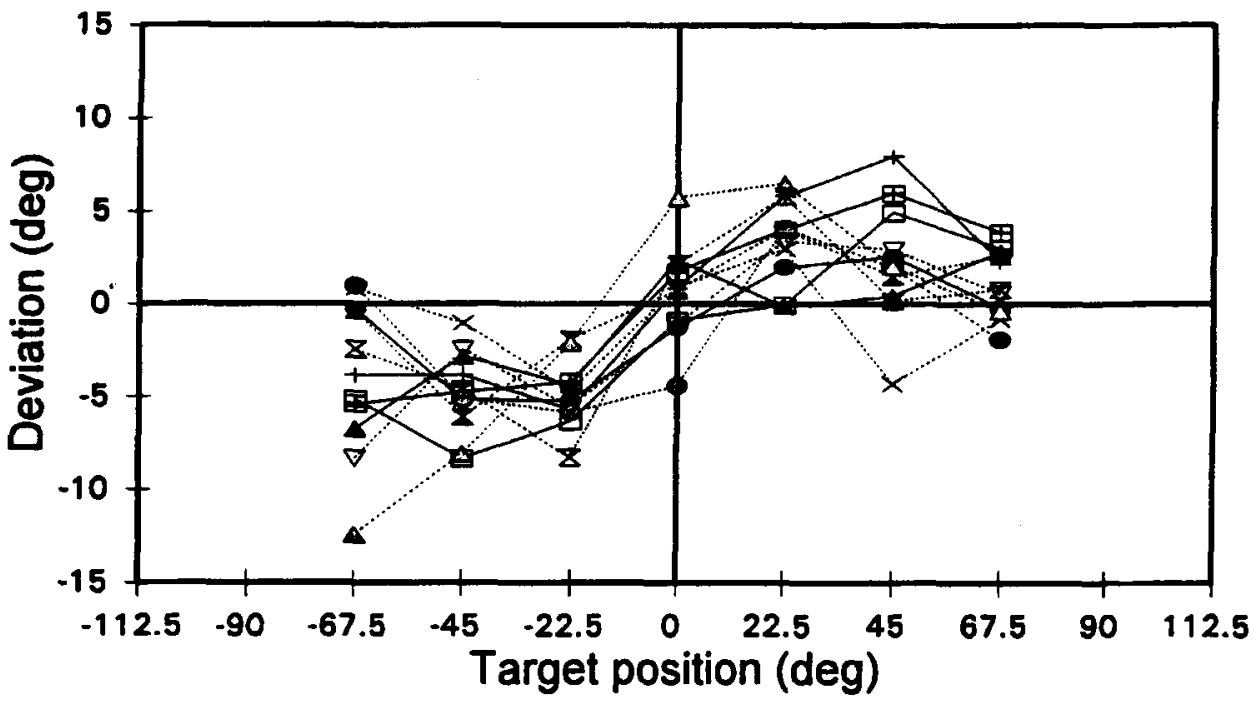

Figure 5. Deviation in initial movement direction as a function of target position. Each symbol represents the mean of four or five trials. The subjects moved only over a distance of $15 \mathrm{~cm}$ toward the target (solid lines; results from Experiment 1) or completed the whole distance (dashed lines; results from de Graaf, Sittig, \& Denier van der Gon, 1994). Note that the deviations in initial movement direction are similar in both conditions. 

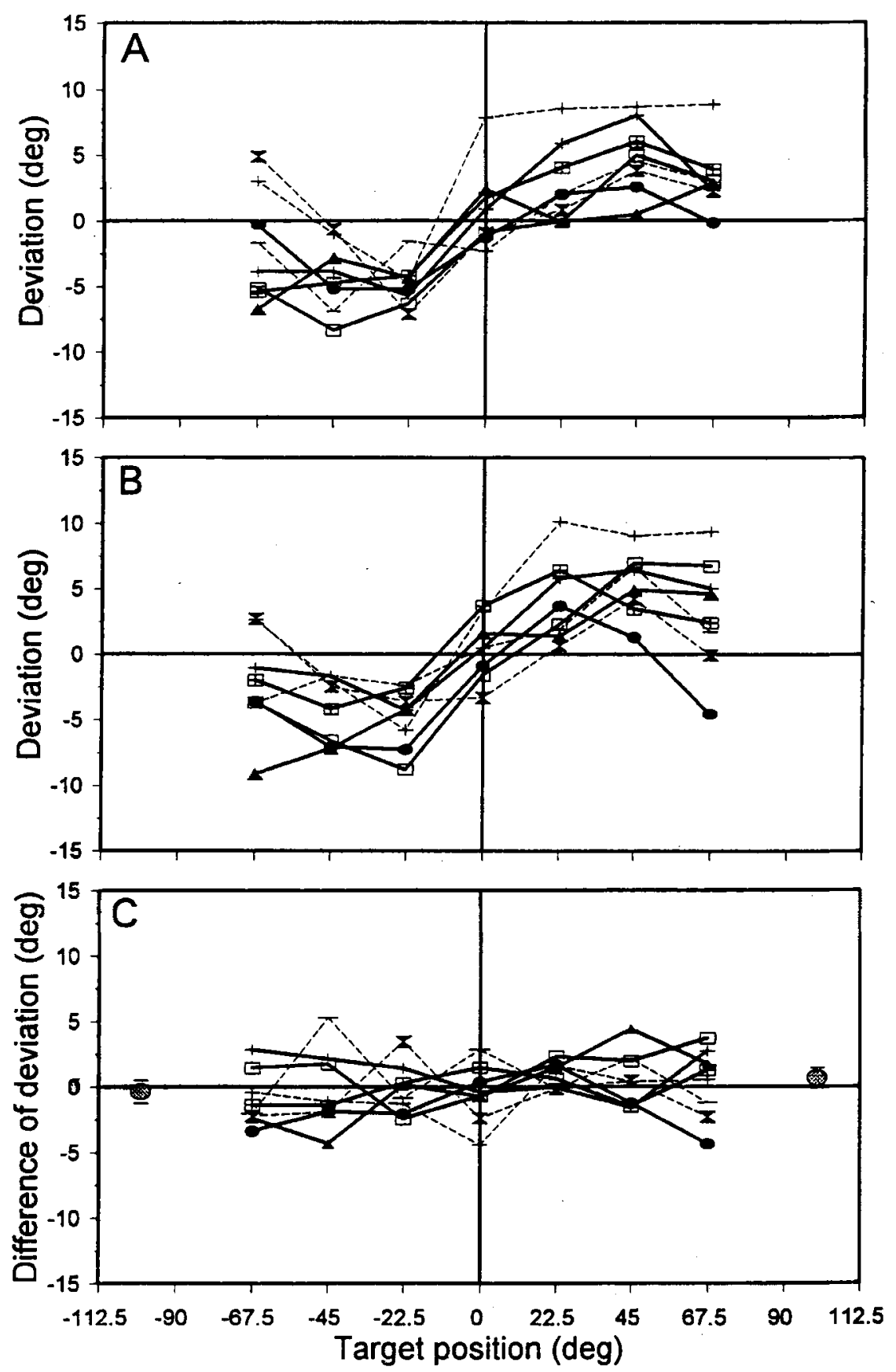

Figure 6. Results of Experiment 1. Deviation in initial movement direction as a function of target position. Each symbol represents the mean of four or five trials. The subjects could see the moving arm (visual feedback; solid lines) or were prevented from seeing the moving arm (no visual feedback; dashed lines). (A) Distance between starting and target position was $31 \mathrm{~cm}$. Mean standard deviation $(S D)$ was $2.9^{\circ}$ for trials where the subjects could see the moving arm and was $4.1^{\circ}$ for trials where they could not. (B) Distance between starting position and target position was $62 \mathrm{~cm}$. Mean $S D$ was $2.9^{\circ}$ for trials where subjects could see the moving arm and was $3.8^{\circ}$ for trials where they could not. (C) Difference between the deviations in initial direction for movements toward the 62-cm targets and the deviations for movements toward the 31-cm targets. The distorted location hypothesis predicts that the difference will be positive for targets in the right half of the stimulus surface and negative for those in the left half. For each side of the stimulus surface, we calculated the mean difference of deviation by averaging over all subjects and the targets at that side. The large dots on the left and right sides of the figure indicate these mean values plus or minus two times the standard error $\left(-0.35^{\circ} \pm 0.9^{\circ}\right.$ for the left side, and $0.66^{\circ} \pm 0.8^{\circ}$ for the right side). 


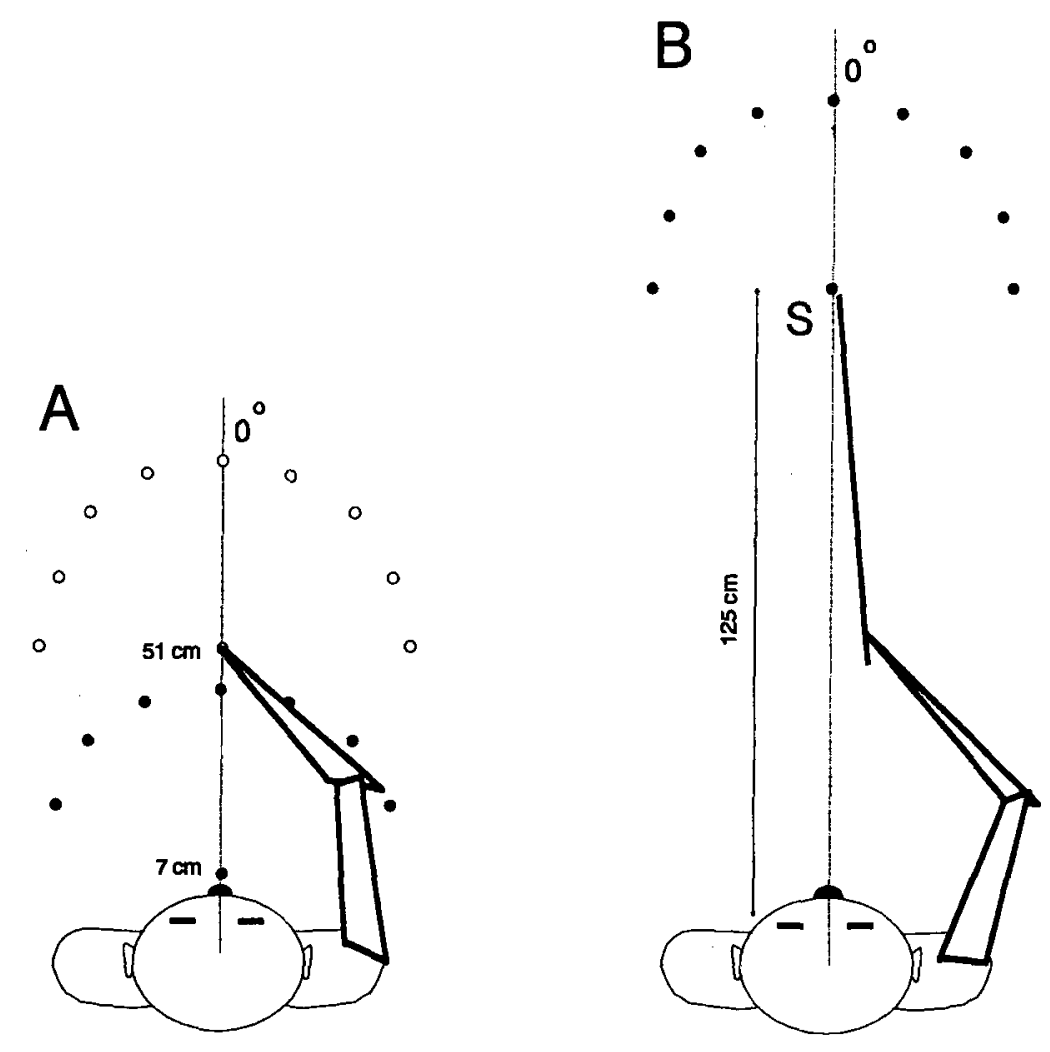

Figure 7. Experimental set-up for Experiment 2. (A) Condition 1. The starting positions are presented at distances of 7 and $51 \mathrm{~cm}$. The target positions, located at $31 \mathrm{~cm}$ from the starting position (S), range from $-6.75^{\circ}$ to $+67.5^{\circ}$ for $\mathrm{S}$ at $7 \mathrm{~cm}$ (black dots) and range from $-90^{\circ}$ to $+90^{\circ}$ for $S$ at $51 \mathrm{~cm}$ (white dots), with intervals of $22.5^{\circ}$. (B) Condition 2. The starting position is presented at a distance of $125 \mathrm{~cm}$. The target positions, located at a distance of $31 \mathrm{~cm}$ from $S$, range from $-90^{\circ}$ to $+90^{\circ}$, with intervals of $22.5^{\circ}$. The subject had a stick in the hand, the tip of which he/she had to move from $S$ toward the target positions. The eyes of the subject were approximately $1 \mathbf{~ m}$ above the stimulus surface in Condition 2.

face and negative for targets on the left side. However, this was clearly not the case. Statistical testing ( $t$ test for paired comparison of sample means) confirmed this observation. For each target position (except the $0^{\circ}$ target, since the distorted location model predicts no influence of distance between the starting position and the target position for this target direction), we tested whether the deviations were larger for targets at a distance of $62 \mathrm{~cm}$. We did not find a significant difference for any of the target positions (for each target position, $p>.05$ ). The large dots on the left and right sides of Figure $6 \mathrm{C}$ show the mean difference (plus or minus two times the standard error) for all subjects and all targets at the left and the right side, respectively, of the stimulus surface. The results show that the increase in deviations with increasing target distance from 31 to $62 \mathrm{~cm}$, if present at all, cannot exceed $1.0^{\circ}$, which is much smaller than a distorted location model (as presented in Figure 2) would predict.

\section{Experiment 2: Varying the Distance Between Starting Position and the Body}

The results of Experiment 1 clearly do not favor the distorted location model (which predicts an increase in the magnitude of the deviations with an increase in target distance), but they do favor the biased direction model. Therefore, in Experiment 2, we explored the biased direction model further by varying the distance of the starting position from the frontal plane of the subject. Note that the biased direction model predicts that the initial movement direction does not depend on the distance between the subject's trunk and the starting position since the target directions are determined relative to the starting position. The distorted location model, however, will in general predict a completely different pattern of initial movement directions.

Experimental set-up. The experiment consisted of two experimental conditions.

Condition 1. Five subjects ( 3 of these subjects had participated in Experiment 1) participated in Condition 1 of Experiment 2. Figure 7A shows the experimental setup. Two starting positions were presented in front of the subject at distances of 7 and $51 \mathrm{~cm}$ from the body. The targets were located at a distance of $31 \mathrm{~cm}$ from the starting position. The subjects could see the moving arm.

Condition 2. Four subjects ( 1 of these subjects also participated both in Condition 1 and in Experiment 1) 
participated in Condition 2 of Experiment 2. Figure 7B shows the experimental set-up. The starting position was presented in front of the subject at a distance of $125 \mathrm{~cm}$ from the body. The targets were located at a distance of $31 \mathrm{~cm}$ from the starting position. The subjects had a stick in the hand, the tip of which they had to move from the starting position in the direction of the target position. The eyes of the subjects were approximately $1 \mathrm{~m}$ above the stimulus surface to ensure that the subjects could see the starting and target positions clearly. The subjects could see the stick during the movements.

Note that, since in Experiment 1 the starting position was presented at a distance of $26 \mathrm{~cm}$, we can now compare the deviations in initial movement direction for four different distances between the starting position and the body of the subject.

Results. Figure 8 shows the mean deviations in initial movement direction for the three distances of the starting positions that were tested in Experiment 2. Figures $8 \mathrm{~A}$ and $8 \mathrm{~B}$ show the results for Condition 1, where the starting position was presented at distances of 7 and $51 \mathrm{~cm}$, respectively. Figure $8 \mathrm{C}$ shows the results for Condition 2 , where the starting position was presented at a distance of $125 \mathrm{~cm}$. When we compare these figures with Figure 6A (where the starting position was presented at a distance of $26 \mathrm{~cm}$ ), it can be seen that the deviations are very much alike for the various distances of the starting position. However, the pattern of the deviations for distances of the starting position of 7 and $51 \mathrm{~cm}$ is somewhat more variable. This might have been caused by the extreme positions in which the subject had to put the arm when the index finger was on these starting positions. The subjects mentioned that in these conditions they were less free to move the arm in the direction they wanted, especially for targets in the right half of the stimulus surface.

For each target position, we statistically compared the magnitude of the deviations for the four distances $(7,26$, 51 , and $125 \mathrm{~cm}$ ) between the starting position and the body. For the starting position at $26 \mathrm{~cm}$, we used the trials where the subjects could see the moving arm. We could not test for each subject individually, because not all subjects participated in all four conditions. Using an analysis of variance ( $F$ test), we found that, for the three targets in the right half of the stimulus surface, the deviations differed significantly between the starting position at a distance of $125 \mathrm{~cm}$ and the starting position at one or two of the other three distances $(p<.05)$. For none of these targets did the deviations for distances of 7,26 , and $51 \mathrm{~cm}$ differ significantly. In the left half of the stimulus surface, we did not find a significant difference in the deviations between any of the four distances of the starting position. It appears that the distance between the starting position and the body does not systematically influence the deviations in initial movement direction. Moreover, we found significant differences only for targets toward which the subject felt less free to move (i.e., targets in the right half of the stimulus surface). Therefore, we believe that these results do not permit us to conclude that the distance between the starting position and the body essentially influences the initial direction of the arm movements.

\section{Experiment 3: Translating the Starting Position to the Right}

The results of Experiment 2 show that, as long as the orientation of the line through starting position and target position does not change relative to a certain reference, the deviations in initial movement direction do not depend on the distance of the starting and target positions from the body. This again supports the biased direction model. In Experiment 3, we investigated what the reference plane is relative to which target direction is estimated. The fact that the deviations in initial movement direction are symmetrical around the saggital axis (straight ahead) of the subjects might suggest to us that the medial plane is the reference plane. However, up to this point, we had only presented the starting position in the medial plane of the subject. Therefore, in Experiment 3, we translated the starting position to the right of the medial plane. Such a change would lead to a completely different pattern of initial movement directions according to the distorted location model. The biased direction model, on the other hand, would predict either no change (if the sagittal plane is the reference plane) or a rotation of the initial movement direction pattern (with a different reference plane).

Experimental set-up. Six subjects participated in two experimental conditions; 3 of these subjects had participated in Experiment 1.

Condition 1. Figure 9A shows the experimental set-up of Condition 1 . One starting position $\left(\mathrm{S}_{1}\right)$ was located in the medial plane of the subject at a radial distance of $26 \mathrm{~cm}$ from the body midline. The other starting position $\left(\mathrm{S}_{2}\right)$ was at the same distance from the body midline but was rotated by $40^{\circ}$ to the right around the body midline. Seven target positions for $S_{1}$ and six target positions for $\mathrm{S}_{2}$ were presented at a distance of $31 \mathrm{~cm}$ from their starting positions. A shield prevented the subjects from seeing the moving arm.

Condition 2. Figure 9B shows the experimental set-up of Condition 2. The starting position was the same as $S_{1}$ in Condition 1, but the arm position was the same as $S_{2}$ in Condition 1. Seven targets were presented at a distance of $31 \mathrm{~cm}$ from the starting position. A lightweight rigid stick was firmly attached to the hand of the subject such that when the index finger was on $S_{2}$, the tip of the stick was at $S_{1}$. The subject could see the stick during the entire movement. The subject was asked not to rotate the wrist while moving the stick. Careful observation showed that the subjects did not rotate the wrist for at least the first $10 \mathrm{~cm}$ of the movement.

Results. The results are shown in Figure 10. Figures $10 \mathrm{~A}$ and $10 \mathrm{~B}$ show the results for Condition 1. In Figure $10 \mathrm{~A}$, it can be seen that, for arm movements started from the medial plane $\left(\mathrm{S}_{1}\right)$ toward the target that was positioned straight ahead $\left(0^{\circ}\right)$, on average, no deviations in initial movement direction occurred. For the other targets, all subjects showed clear deviations from target direction, away from the medial plane. 

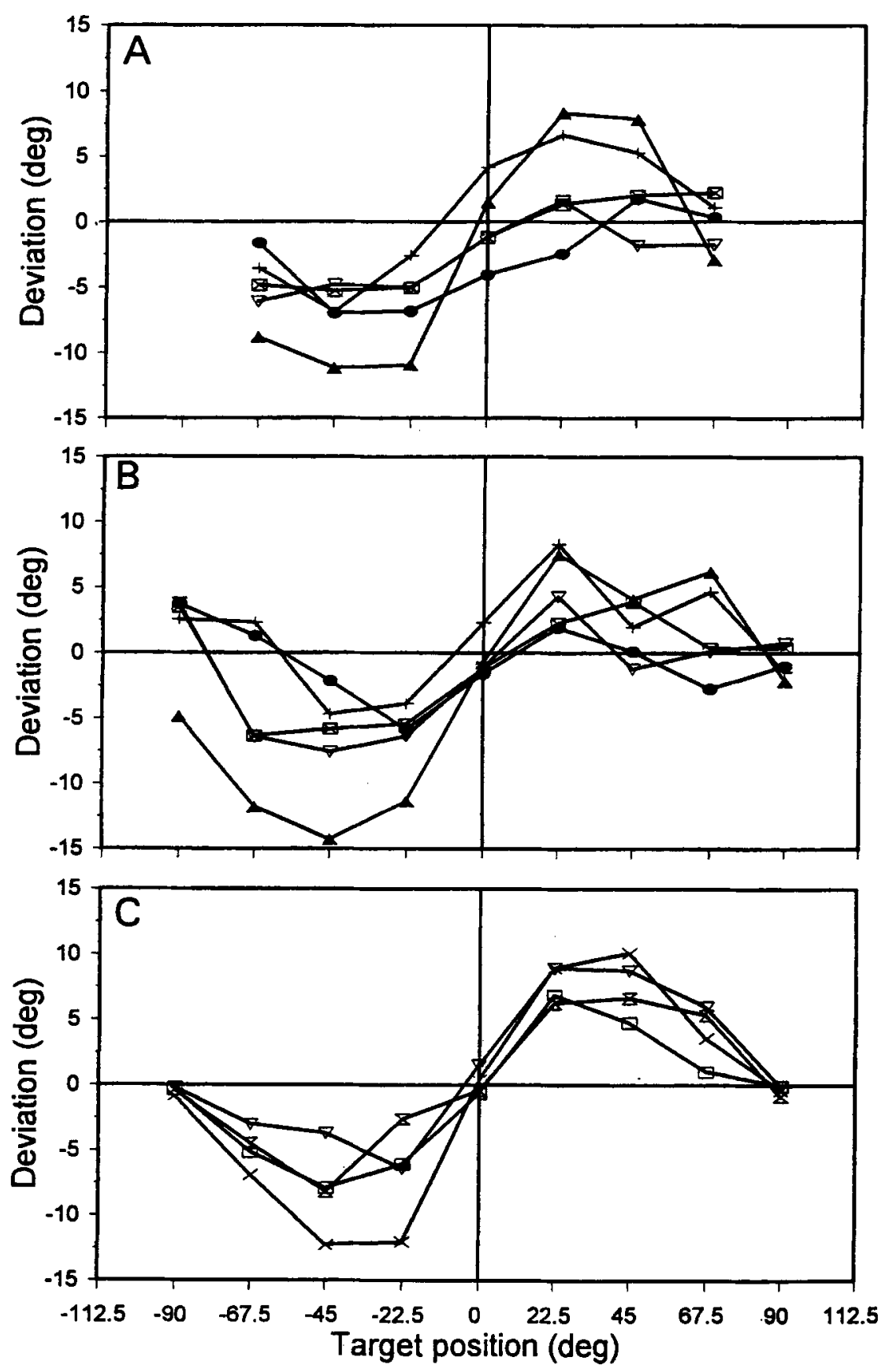

Figure 8. Results of Experiment 2. Deviation in initial movement direction for three distances of the starting position from the body. For the subjects who also participated in Experiment 1, the same symbols as those in Figure 6 are used. (A) Distance of the starting position from the body was $7 \mathrm{~cm}$. Mean $S D$ was 3.0 $0^{\circ}$. (B) Distance of the starting position from the body was $51 \mathrm{~cm}$. Mean $S D$ was $2.9^{\circ}$. (C) Distance of the starting position from the body was $125 \mathrm{~cm}$. Mean $S D$ was $3.9^{\circ}$.

Figure 10B shows the results for the movements from the starting position that was located to the right of the medial plane $\left(\mathrm{S}_{2}\right)$. It can be seen that the deviations in initial movement direction were negative for movements toward most targets, except for the $45^{\circ}$ (and possibly the $22.5^{\circ}$ ) target. Compared with Figure 10A, the pattern of deviations in Figure 10B seems to be translated to the right. It appears that the symmetry axis of the deviations is between the $22.5^{\circ}$ and $45^{\circ}$ target. Note that this is approximately the direction in which the starting position was located with respect to the body midline of the subject (indicated by the dashed line in Figure 10B). This shift of the symmetry axis when $S$ is translated cannot be caused by the subject's opportunity to visually align the starting position and the target that is located in the same line of sight as the starting position, since a shield pre- 

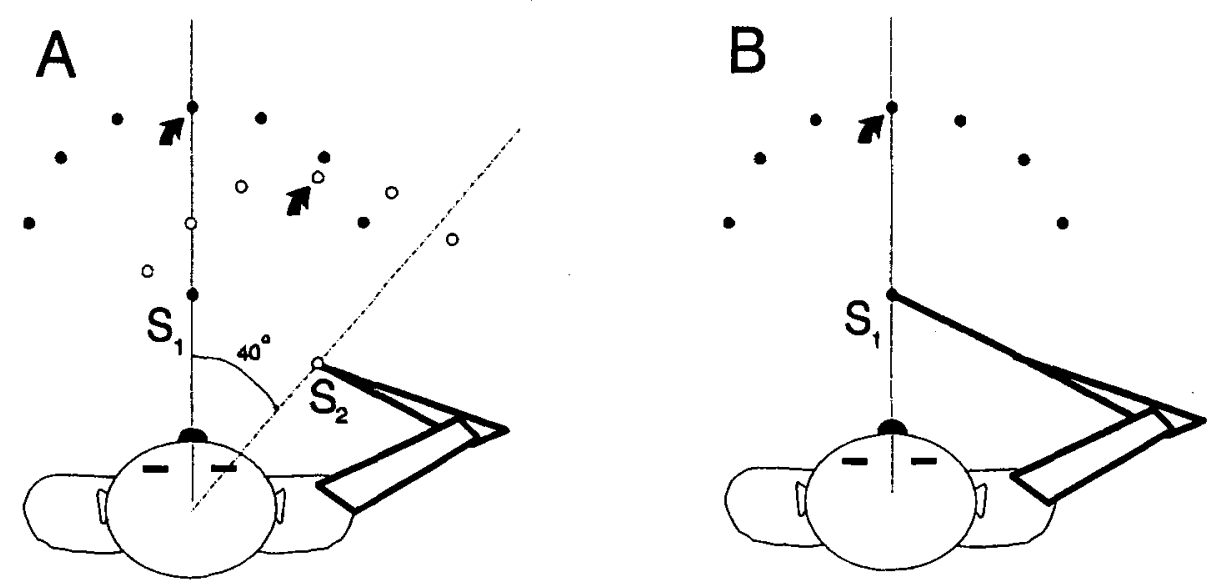

\begin{abstract}
Figure 9. Experimental set-up for Experiment 3. (A) Condition 1. Two starting positions $S_{1}$ and $S_{2}$ were presented at a distance of $26 \mathrm{~cm}$ from the body midline of the subject. Black dots represent target positions that were presented when $S_{1}$ was presented; open circles represent target positions when $S_{2}$ was presented. The target positions ranged from $-67.5^{\circ}$ to $+67.5^{\circ}$ for $S_{1}$ and from $-67.5^{\circ}$ to $45^{\circ}$ for $S_{2}$, with intervals of $22.5^{\circ}$. The distance between starting position and target position was $31 \mathrm{~cm}$. (B) Condition 2. $S_{1}$ was presented at the same location as $S_{1}$ in Condition 1 (see Panel $A$ ). A stick was attached to the subject's hand such that when the tip of the stick was at $S_{1}$, the arm position was the same as when the subject had the index finger on $\mathrm{S}_{2}$ in Condition 1 . Arrows indicate $0^{\circ}$ target in both conditions.
\end{abstract}

vented the subjects from seeing the starting position. This shield was placed before the target appeared.

One might object that, in translating the location of the starting position, one also changes the position of the arm. This change in arm position might have changed the pattern of the deviations. Although we already argued in the introduction of this paper that biomechanical factors are highly unlikely to cause the deviations in initial movement direction, we again tested this. In Condition 2, we changed arm position, but not starting position: the subject had a stick attached to the hand such that when the tip of the stick was at $S_{1}$, the arm position was the same as if he/she had the index finger on $S_{2}$. If the initial movement direction is indeed influenced by actual arm position (i.e., by mechanical factors influencing the execution of the movement), we now expect to find a symmetry axis at roughly target $40^{\circ}$ (as in Condition 1 for $S_{2}$ ).

Figure $10 \mathrm{C}$ shows the results. It appears that the deviations are now symmetrical around the $0^{\circ}$ target. Note that this is the same as in Figure 10A. The magnitude of the deviations is somewhat smaller than in Figure 10A, but this may be related to the fact that, in this experimental condition, the subjects could see the stick during the movements, whereas, in Condition 1, they could not see the arm. This result clearly shows that the shift in the pattern of the initial movement directions we found in Condition 1 was caused purely by the shift of the starting position relative to the medial plane of the subject and not by the change of arm position.

\section{DISCUSSION}

This study was performed to investigate what mechanism underlies the biased estimate of target direction found in previous studies (de Graaf et al., 1991, 1994; Hollins \&
Kelley, 1988; Sittig \& de Graaf, 1994). The results of the three experiments show that the deviations in initial movement direction depend on the orientation of the line through starting position and target position but not on the distance between the starting position and the target. These results strongly support the biased direction model.

First of all, the results of Experiment 1 show that the magnitude of the deviations does not depend on the distance between starting position and target position. As we prove in the Appendix, the distorted location model predicts an increase in the magnitude of the deviations with increasing distance between starting position and target position. This proof is based on the assumption that straight lines in the real world are transformed into curves without a point of inflection in the internal representation. We feel this assumption is reasonable. For example, in the case of a contraction model, this means that the magnitude of the contraction increases monotonically with distance from the body. However, the distorted location model can be rejected even if one does not want to accept this assumption: If the model were to hold, it would be an extremely strong coincidence that we find approximately equal deviations for both tested target distances for all target directions. Namely, finding similar deviations for two targets that are located on the same straight line through the starting position in the real world would imply that, in the internal representation, the transformed straight line has one or more points of inflection somewhere between the two internally represented target positions, such that the nonlinear effects are exactly canceled out. It is highly unlikely that we would find this for all target directions, since this would require a rather special transformation of locations. Therefore, we believe that the distorted location model cannot account for the biased estimate. 

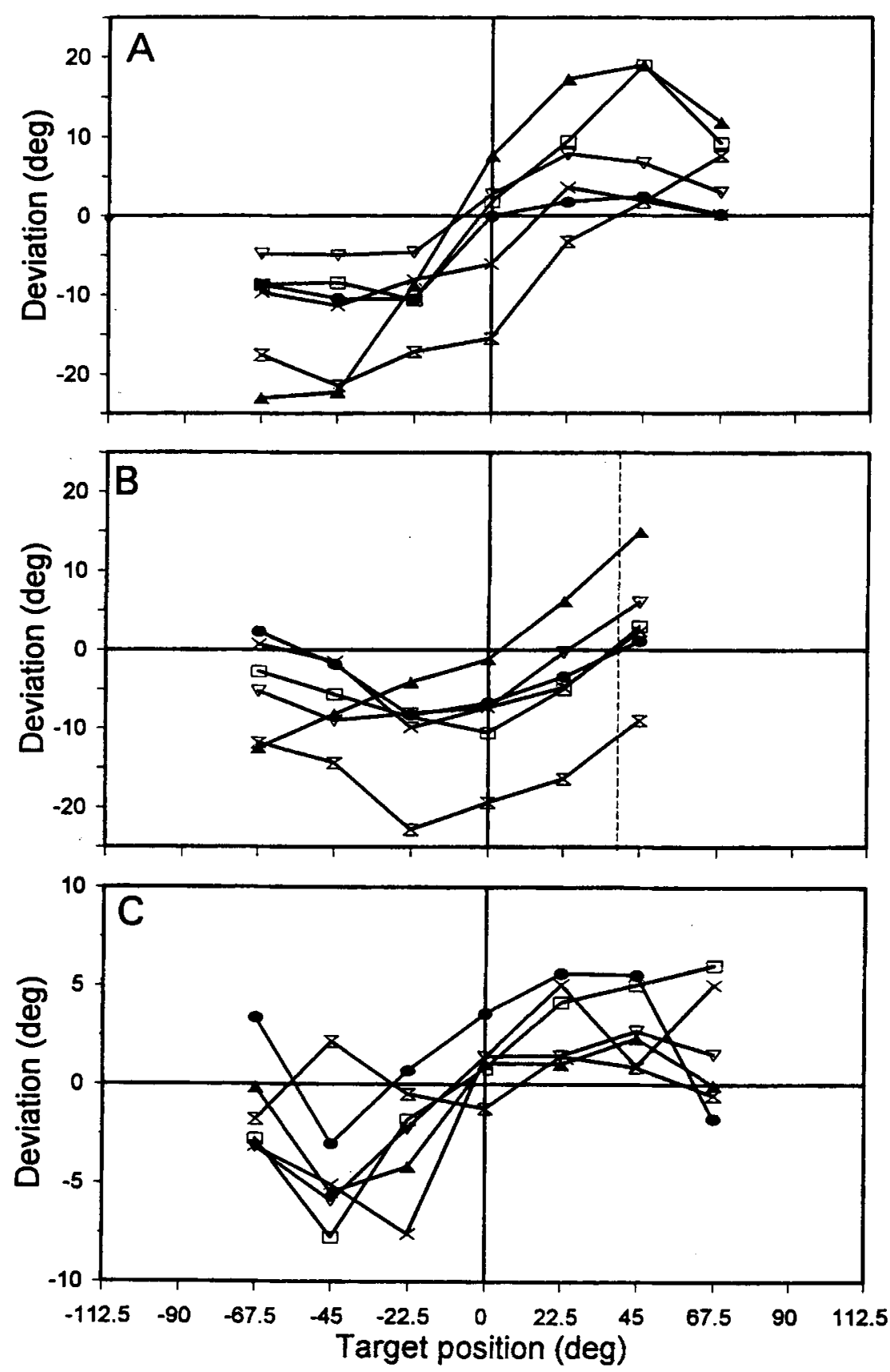

Figure 10. Results of Experiment 3. Deviation in initial movement direction as a function of target position. Each symbol represents the mean of four or five trials. For the subjects who also participated in Experiments 1 and 2, the same symbols as those in Figures 6 and 8 are used. (A) Deviations in initial direction for movements that started at $S_{1}$ (see Figure 9A). Mean $S D$ was $4.3^{\circ}$. (B) Deviations for movements that started at $S_{2}$. The dashed line indicates the direction of the line through body midline and $S_{2}$. Note that the deviations were, on average, zero for this direction. Mean $S D$ was $4.3^{\circ}$. (C) Deviations in initial movement direction as found in Condition 2, where the subject had a stick attached to the hand (see Figure 9B). Mean $S D$ was $4.3^{\circ}$. Note that the symmetry axis of the deviations is comparable to that in Panel $A$.

Furthermore, the finding that the distance between the whole stimulus configuration and the body of the subject does not systematically influence the estimate of target direction also supports the biased direction model. If the distorted location model were to hold, one would expect the deviations to depend on the distance between the stimulus configuration and the body of the subject. ${ }^{2}$ The biased direction model, on the other hand, predicts similar deviations for varying distances between the starting position and the body, since the orientation of the line through 
starting position and target position does not change when the stimulus configuration is located at different distances from the body of the subject. Therefore, the results of Experiments 1 and 2 strongly suggest that the deviations in initial movement direction are caused not by a distorted internal representation of locations but by a biased estimate of target direction relative to a reference. The results of Experiment 3 suggest that this reference relative to which target direction is estimated is the plane through longitudinal body or head axis and starting position.

The results of the present study are relevant for the discussion about how we code the displacement of our limbs in space. It now seems well accepted that we plan a limb movement in space coordinates (Hogan, 1988; Morasso, 1981). However, it is still an open question as to what type of coding is used in the planning and execution of goaldirected movements. Two different coding schemes have been suggested: displacement could be coded in terms of locations within an internal space map (locus calibration) or in terms of direction and distance (vectorial coding) (Paillard, 1991a). In the literature, one can find support for the locus calibration hypothesis (e.g., Flanagan, Feldman, \& Ostry, 1992) and for the vector coding hypothesis (e.g., Bock \& Eckmiller, 1986). We show here that neither target distance nor the actual location of starting position and target position relative to the body of the subject are relevant for the initial direction of the movements (as long as target direction relative to the reference plane is the same). This strongly suggests that the goal-directed movements in the present study were planned in terms of target direction and not in terms of target location, which supports the vector coding hypothesis. The mechanism of deducing directional information seems to be a scaleindependent process in which distance between the whole stimulus configuration and the body and distance between starting position and target position do not influence the direction estimate of the target relative to the starting position. In a three-dot alignment task, Sittig and de Graaf (1994) showed that in the frontoparallel plane - even for distances of only $2.5^{\circ}$ visual angle between the two extreme dots-similar deviations occur.

The hypothesis that a target is represented in terms of direction (and distance) relative to the starting position is supported by neurophysiological data. In the oculomotor system-more specifically, in the intermediate (Sparks, 1991) and deep (Schlag-Rey, Schlag, \& Shook, 1989) layers of the superior colliculus and in the frontal eye fields (Goldberg \& Bruce, 1990)-it has been demonstrated that a target is coded as a vector. There is also neurophysiological support for arm movements. In the motor cortex (Georgopoulos, Kettner, \& Schwartz, 1988; Kettner, Schwartz, \& Georgopoulos, 1988; Schwartz, Kettner, \& Georgopoulos, 1988), the premotor cortex (Caminiti, Johnson, Galli, Ferraina, \& Burnod, 1991), and parietal cortex area 5 (Kalaska, 1988), it has been found that the direction of an upcoming arm movement is coded in the population discharge of directional tuned cortical cells. Kalaska, Cohen, Prud'homme, and Hyde (1990) showed that, in parietal area 5, this neuronal ac- tivity depends not on the movement dynamics but solely on the movement kinematics. These results support the notion of vector coding of the target position relative to the starting (and, thus, hand) position.

As mentioned above, in the literature, one can also find evidence for the hypothesis that we code the displacement of our limbs as successive locations in space (e.g., Van Sonderen \& Denier van der Gon, 1990, or the equilibrium point model of Flanagan et al., 1992). This seems to contradict our findings. However, these authors had their subjects perform very fast arm movements. In our study, the movements were performed very slowly $(3-6 \mathrm{~cm} / \mathrm{sec})$. Note that, in the study of Bock and Eckmiller (1986) (of which the results are in favor of the vector coding hypothesis), the subjects moved at work pace, not maximally fast. This suggests that the coding used in slow and intermediate-fast movements differs from that used in very fast movements. Indeed, it has been shown that the coding scheme might differ according to the specific demands of the task (Abrams \& Landgraf, 1990; Mack, Heuer, Villardi, \& Chambers, 1985).

Finally, we would like to discuss the reference system used in representing a target position. It has been suggested that the center of the reference frame is located between the body midline of the subject and the shoulder of the moving arm (Flanders, Helms Tillery, \& Soechting, 1992; Soechting \& Flanders, 1989). This does not seem to be in agreement with our finding that target direction estimation is related to a reference plane through the longitudinal head or body axis. However, these authors came to their conclusion by analyzing end position errors of arm movements. As these authors also mention (Flanders et al., 1992, p. 319), it is not at all certain that the process of deducing directional information at the start of a movement needs to have the same center of reference as the homing-in phase of the movement; the latter could be an entirely different process in which different information is used. We would like to add here that this might especially hold for slow movements, in which there is ample time for homing in at the end of the movement. Second, the authors have not varied the initial position of the arm movements. As Massone (1992) pointed out in the open peer commentary of the target article of Flanders et al. (1992), it is known that the starting position of the hand influences the errors at the end of a movement.

In the literature, a distinction is made between an egocentric representation and an allocentric representation of a target position (Blouin et al., 1993; Paillard, 1991b). An egocentric representation means a representation of a target relative to the head or trunk of the subject. An allocentric representation of a target is a representation relative to a stable visual landmark (Blouin et al., 1993). Our results now support the hypothesis that a target is represented relative to the starting (and, thus, hand) position. However, this form of representation is incorporated in neither of the two representations. First, it is not egocentric, since it is not a representation relative to the trunk or head. Second, since we showed that visual information is not relevant for the initial direction of the 
movement (de Graaf et al., 1994), it is not a representation relative to a visual landmark. It appears that the currently available theories about reference frames are not sufficient to explain the data of the present study.

The present study does not enable us to draw conclusions about the process of target direction estimation, nor can we say anything about the possible cause of these biases in target direction estimation. As we argued in the introduction and again confirmed in Experiment 3, biomechanical constraints experienced during the performance of the movements are highly unlikely to be responsible for the deviations in initial movement direction. However, we cannot exclude that the process of estimating the target direction is influenced by the perceived ease of making specific movements: The internal representation of target direction might be modified to compensate for the perceived biomechanical difficulties of particular movements. The fact that, in Experiment 2, we found the results to be influenced by biomechanical constraints supports this idea. However, we want to stress that the demonstrated biases are not related in a simple way to the active exploration of space, because even when starting and target positions are located out of grasping space (as is the case for a starting position at a distance of $125 \mathrm{~cm}$ ), similar deviations occur.

Whatever the cause of the demonstrated biases, they did enable us to investigate the process of spatial localization in the planning of goal-directed movements. We conclude that the initial direction of slow goal-directed movements is planned on the basis of a scale-independent (biased) estimate of target direction, not of target location. This supports the hypothesis that we code the displacement of our limbs in terms of a vector.

\section{REFERENCES}

Abrams, R. A., \& Landgraf, J. Z. (1990). Differential use of distance and location information for spatial localization. Perception \& Psychophysics, 47, 349-359.

Blouin, I., Bard, C., Teasdale, N., Palllard, J., Fleury, M., ForGET, R., \& LAMARRE, Y. (1993). Reference systems for coding spatial information in normal subjects and a deafferented patient. $E x$ perimental Brain Research, 93, 324-331.

BOCK, O., \& ECKMILLER, R. (1986). Goal-directed arm movements in the absence of visual guidance: Evidence for amplitude rather than position control. Experimental Brain Research, 62, 451-458.

Caminiti, R., Johnson, P. B., Galli, C., Ferraina, S., \& Burnod, Y. (1991). Making arm movements within different parts of space: The premotor and motor cortical representations of a coordinate system for reaching to visual targets. Journal of Neuroscience, 11, 1182-1197.

DE GRAAF, J. B. (1994). The initial direction of slow goal-directed arm movements: Deviations as a means to study spatial representation. Unpublished doctoral thesis, Delft University of Technology, Delft, The Netherlands.

de Graaf, J. B., Sittig, A. C., \& Denier van der Gon, J. J. (1991). Misdirections in slow goal-directed arm movements and pointersetting tasks. Experimental Brain Research, 84, 434-438.

de GraAf, J. B., Sittig, A. C., \& Denier van der Gon, J. J. (1993, September). How do misdirections in slow arm movements depend on location of starting and target position? Abstract for the 16th Annual Meeting of the European Neuroscience Association (p. 280). Madrid.

de Graaf, J. B., Sittig, A. C., \& Denier van der Gon, J. J. (1994). Misdirections in slow goal-directed arm movements are not primarily visually based. Experimental Brain Research, 99, 464-472.

Flanagan, J. R., Feldman, A. G., \& Ostry, D. J. (1992). Equilibrium trajectories underlying rapid target-directed arm movements. In G. E. Stelmach \& J. Requin (Eds.), Tutorials in motor behavior II (pp. 661-675). Amsterdam: Elsevier.

Flanders, M., Helms Tillery, S. I., \& Soechting, J. F. (1992). Early stages in a sensorimotor transformation. Behavioral \& Brain Sciences, 15, 309-362.

Georgopoulos, A. P., Kettner, R. E., \& Schwartz, A. B. (1988). Primate motor cortex and free arm movements to visual targets in three-dimensional space: II. Coding of the direction by a neuronal population. Journal of Neuroscience, 8, 2928-2937.

GoldberG, M. E., \& BrUCE, C. J. (1990). Primate frontal fields: III. Maintenance of a spatially accurate saccade signal. Journal of Neurophysiology, 64, 489-508.

HoGAN, N. (1988). Planning and execution of multijoint movements. Canadian Journal of Physiology \& Pharmacology, 66, 508-517.

Hollins, M., \& KELIEY, E. K. (1988). Spatial updating in blind and sighted people. Perception \& Psychophysics, 43, 380-388.

KALASKA, J. F. (1988). The representation of arm movements in postcentral and parietal cortex. Canadian Journal of Physiology \& Pharmacology, 66, 455-463.

Kalaska, J. F., Cohen, D. A. D., Prud'homme, M., \& Hyde, M. L. (1990). Parietal area 5 neuronal activity encodes movement kinematics, not movement dynamics. Experimental Brain Research, 80, 351-364.

Kettner, R. E., Schwartz, A. B., \& Georgopoulos, A. P. (1988). Primate motor cortex and free arm movements to visual targets in three-dimensional space: III. Positional gradients and population coding of movement direction from various movement origins. Journal of Neuroscience, 8, 2938-2947.

Mack, A., Heuer, F., Villardi, K., \& Chambers, D. (1985). The dissociation of position and extent in Müller-Lyer figures. Perception \& Psychophysics, 37, 335-344.

MAssone, L. L. E. (1992). Coordinate transformations: Some basic questions. Behavioral \& Brain Science, 15, 345-346.

Morasso, P. (1981). Spatial control of arm movements. Experimental Brain Research, 42, 223-227.

Paillard, J. (1991a). Knowing where and knowing how to get there. In J. Paillard (Ed.), Brain and space (pp. 461-481). Oxford: Oxford University Press.

PAILlARD, J. (1991b). Motor and representational framing of space. In J. Paillard (Ed.), Brain and space (pp. 163-182). Oxford: Oxford University Press.

Schlag-Rey, M., Schlag, J., \& Shook, B. (1989). Interactions between natural and electrically evoked saccades: I. Differences between sites carrying retinal error and motor error signals in monkey superior colliculus. Experimental Brain Research, 76, 537-547.

Schwartz, A. B., KetTner, R. E., \& Georgopoulos, A. P. (1988). Primate motor cortex and free arm movements to visual targets in three-dimensional space: I. Relations between single cell discharge and direction of movement. Journal of Neuroscience, 8, 2913-2927.

SitTiG, A. C., \& DE GraAF, J. B. (1994). Orientation dependent misalignments in a visual alignment task. Vision Research, 34, 2195-2203.

Soechting, J. F., \& Flanders, M. (1989). Sensorimotor representation for pointing to targets in three-dimensional space. Journal of Neurophysiology, 62, 582-594.

SPARKs, D. J. (1991). The neural encoding of the location of targets for saccadic eye movements. In J. Paillard (Ed.), Brain and space (pp. 319). Oxford: Oxford University Press.

Van Sonderen, J. F., \& Denier van der Gon, J. J. (1990). A simulation study of centrally programmed fast two-joint arm movements: Responses to single- and double-step target displacements. Biological Cybernetics, 63, 34-44.

Vincken, M., \& Denier VAN der Gon, J. J. (1985). Stiffness as a control variable in motor performance. Human Movement Science, 4, 307-319.

\section{NOTES}

1. Part of the results have been published in the form of an abstract (de Graaf et al., 1993). Partial results of Experiment 3 have been published in de Graaf et al. (1994). 
2. A general statement is not possible, since the deviations depend on the exact transformation one chooses. In the case of an exponential contraction, the deviations will not depend on the distance between the starting position and the body, but then the deviations will depend on the distance between the starting position and the target position in such a way that doubling the target distance would about double the magnitude of the deviations. This is clearly not what we found: We showed in Experiment 1 that doubling the target distance did not result in $a \sim 4^{\circ}$ increase of the deviations. No significant increase was found; we calculated that the increase, if present at all, could not exceed $1.5^{\circ}$ (see Figure 6)

\section{APPENDIX}

Let $\Phi: \mathbb{R}^{2} \rightarrow \mathbb{R}^{2}$ be a nonlinear transformation of locations from the real world (rw) to the internal representation (ir). We assume that $\Phi$ is bijective and continuously differentiable. Let $S_{\mathrm{rW}}$ and $T_{\mathrm{rw}}$ be the starting position and the target position, respectively, in the real world, and let $S_{\mathrm{ir}}$ and $T_{\mathrm{ir}}$ be their $\Phi$-images in the internal representation. The initial movement direction in the real world is the direction at $S_{\mathrm{rw}}$ of the inverse transformation of $\Phi$ of the straight line through $S_{\mathrm{ir}}$ and $T_{\mathrm{ir}}$ in the internal representation. Since $\Phi$ is a nonlinear transformation, the initial movement direction will deviate from target direction.

Concerning the transformation $\Phi$, we make the following two assumptions:

Assumption 1. Let $P_{r w}$ be a point in the real world. Let

$$
\gamma(t)=(1-t) S_{\mathrm{rw}}+t P_{\mathrm{rw}}
$$

be a parametrization of the line through $S_{\mathrm{rw}}$ and $P_{\mathrm{rw}}$. Let $d(P, Q), P, Q \in \mathbb{R}^{2}$ be the Euclidean distance between $P$ and $Q$. We now assume that

$$
d\left(\Phi\left(\gamma\left(t_{2}\right)\right), S_{\mathrm{ir}}\right)>d\left(\Phi\left(\gamma\left(t_{1}\right)\right), S_{\mathrm{ir}}\right), \forall 0<t_{1}<t_{2} .
$$

This means that the $\Phi$-image of $\gamma\left(t_{1}\right)$ is closer to $S_{\mathrm{ir}}$ than is the $\Phi$-image of $\gamma\left(t_{2}\right)$ whenever $0<t_{1}<t_{2}$. (Note that one can, in fact, prove this assumption if one takes into account that the movement trajectories have to be straight lines for the $0^{\circ}$ and $\pm 90^{\circ}$ targets.)

Assumption 2. We assume that the $\Phi$-image of a straight line through $S_{\mathrm{rw}}$ and $T_{\mathrm{rw}}$ does not contain a point of inflection. (For a discussion of this assumption, please refer to the Discussion section of this paper.)

We will now deduce the requirement that $\Phi$ must satisfy in order to predict the deviations in initial movement direction that we found (i.e., deviations from target direction away from the medial plane; see Figure 1). Let the $x$-axis be a line parallel to the frontal plane of the subject, and let the $y$-axis be the sggital axis (straight ahead) of the subject.

Definition. Let $P \in \mathbb{R}^{2}$. The angle between $P$ and the positive $y$-axis will be denoted by $\operatorname{Arg}(P)$. Of course, if $P=\left(x_{1}, y_{1}\right)$ then $\operatorname{Arg}(P)=\arctan x_{1} / y_{1}$ for $y_{1}>0$. Given $\Phi, S_{\mathrm{rw}}$, and $T_{\mathrm{rw}}$, the initial movement direction can now be computed as

$$
\operatorname{Arg}\left(J_{S_{\mathrm{rw}}}^{-1}\left(T_{\mathrm{ir}}-S_{\mathrm{ir}}\right)\right)
$$

where

$$
J_{S_{\mathrm{rw}}}=\left(\begin{array}{cc}
\frac{\delta \Phi_{1}}{\delta x} & \frac{\delta \Phi_{1}}{\delta y} \\
\frac{\delta \Phi_{2}}{\delta x} & \frac{\delta \Phi_{2}}{\delta y}
\end{array}\right)_{S_{\mathrm{rw}}}
$$

is the Jacobian of $\Phi$ in $S_{\mathrm{rw}}$, and $\Phi_{1}$ and $\Phi_{2}$ are the coordinate functions of $\Phi$-that is, $\Phi(x, y)=\left(\Phi_{1}(x, y), \Phi_{2}(x, y)\right)$. Note that the initial movement direction is now indicated as the angle with the positive $y$-axis.
Given $S_{\mathrm{rw}}$ located on the $y$-axis and $T_{\mathrm{rw}}$ located in the first quadrant, the transformation $\Phi$ must satisfy the following condition in order to predict deviations from target direction, away from the medial plane:

$$
\operatorname{Arg}\left(T_{\mathrm{rw}}-S_{\mathrm{rw}}\right)<\operatorname{Arg}\left(J_{S_{\mathrm{rw}}}^{-1}\left(T_{\mathrm{ir}}-S_{\mathrm{ir}}\right)\right)
$$

We will show that, from this requirement and the two assumptions stated above, it follows that the deviations in initial movement direction increase for increasing distance between $S_{\mathrm{rw}}$ and $T_{\mathrm{rw}}$. Let $P_{\mathrm{rw}}$ be a point located in the first quadrant. Let $T_{\mathrm{rw}}(t)=$ $(1-t) S_{\mathrm{rw}}+t P_{\mathrm{rw}}$ be a parametrization of the line through $S_{\mathrm{rw}}$ and $P_{\mathrm{rw}}$. We denote the predicted initial direction for movements from $S_{\mathrm{rw}}$ to $T_{\mathrm{rw}}(t)$ by $\alpha(t)$, for $t>0$. We have seen that

$$
\alpha(t)=\operatorname{Arg}\left(J_{S_{\mathrm{rw}}}^{-1}\left(T_{\mathrm{ir}}(t)-S_{\mathrm{ir}}\right)\right) .
$$

We will now prove that $\alpha(t)$ is strictly increasing for $t>0$ (i.e., $\alpha(t)$ is increasing if the distance between target $T_{\mathrm{rw}}$ and starting position $S_{\mathrm{rw}}$ is increasing). Let $\beta(t)$ be the argument of the straight line through $S_{\mathrm{ir}}$ and $T_{\mathrm{ir}}$. That is,

$$
\beta(t)=\operatorname{Arg}\left(T_{\mathrm{ir}}(t)-S_{\mathrm{ir}}\right) .
$$

It suffices to prove that $\beta(t)$ is monotonous for $t>0$. That this is indeed sufficient follows from the following two arguments. First, if $\beta(t)$ is monotonous, then $\alpha(t)$ is also monotonous, since $J_{S_{\mathrm{rW}}}^{-1}$ is an invertible linear mapping. Second, $\alpha(t)$ is decreasing for $t \downarrow 0$. This follows from the following lemma.

LEMMA. $\alpha(t)>\operatorname{Arg}\left(T_{\mathrm{rW}}(t)-S_{\mathrm{rW}}\right)$, and $\alpha(t) \rightarrow \operatorname{Arg}\left(T_{\mathrm{rW}}(t)-\right.$ $S_{\mathrm{rw}}$ ) when $t \downarrow 0$.

Proof. That $\alpha(t)>\operatorname{Arg}\left(T_{\mathrm{rw}}(t)-S_{\mathrm{rW}}\right)$ follows directly from Equation A1. If $t \downarrow 0$, then $\Phi\left(T_{\mathrm{rw}}(t)\right) \rightarrow S_{\mathrm{ir}}$ along the curve $\Phi\left(T_{\mathrm{rw}}\right)$. Therefore, the unit vector

$$
u(t)=\frac{T_{\mathrm{ir}}(t)-S_{\mathrm{ir}}}{\left\|T_{\mathrm{ir}}(t)-S_{\mathrm{ir}}\right\|}
$$

tends to a tangent to $\Phi\left(T_{\mathrm{rw}}\right)$ in $S_{\mathrm{ir}}$, which is

$$
\frac{J_{S_{\mathrm{rw}}}\left(T_{\mathrm{rw}}-S_{\mathrm{rw}}\right)}{\left\|J_{S_{\mathrm{rw}}}\left(T_{\mathrm{rw}}-S_{\mathrm{rw}}\right)\right\|} .
$$

Thus, $J_{S_{\mathrm{rw}}}^{-1} \circ u(t)$ tends to

$$
\frac{T_{\mathrm{rw}}-S_{\mathrm{rw}}}{\left\|J_{S_{\mathrm{rw}}}\left(T_{\mathrm{rw}}-S_{\mathrm{rw}}\right)\right\|} .
$$

Therefore, $\alpha(t)=\operatorname{Arg}\left(J_{S_{\mathrm{rW}}}^{-1}\left(T_{\mathrm{ir}}(t)-S_{\mathrm{ir}}\right)\right) \rightarrow \operatorname{Arg}\left(T_{\mathrm{rW}}-S_{\mathrm{rW}}\right)$, if $t \downarrow 0$.

That $\beta(t)$ is monotonous follows directly from Assumptions 1 and 2 .

We have now proved that, for a model in which the initial movement direction is the transformed direction of the internal straight line at the starting position, and given Assumptions 1 and 2 stated above, any transformation between rw and ir that satisfies the requirement of Equation A1 predicts that the deviations in initial movement direction will increase with increasing distance between the starting position and the target position.

(Manuscript received April 7, 1994; revision accepted for publication August 8,1995 .) 